\title{
DE RELATOS Y EXPERIENCIAS. ELEGIR LA UNIVERSIDAD PARA FORMARSE COMO PROFESOR DE LA ESCUELA SECUNDARIA
}

\author{
Andrea Iglesias* \\ Universidad de Buenos Aires, Argentina \\ andreaiglesias.tics@gmail.com
}

Recibido: 31/10/2018 Aceptado: 25/02/2019

\section{Resumen}

El presente trabajo se desprende de los resultados de mi tesis doctoral donde abordé la relación entre las trayectorias de formación docente y las estrategias de inserción laboral de los profesores principiantes de escuelas secundarias de la Ciudad Autónoma de Buenos Aires.

Me propongo analizar aquí específicamente el relato sobre las experiencias en el trayecto formativo pedagógico de un conjunto de entrevistados que cursaron las carreras de Historia, Geografía y Letras en la Universidad. Posicionados desde el paradigma interpretativo y una perspectiva histórico-cultural, analizamos de qué manera el origen bifronte de la formación docente argentina fue dibujando particularidades en el trayecto pedagógico que realizan quienes eligen la Universidad para formarse como profesores de escuela secundaria.

Los resultados indican que la formación pedagógica recibida por los universitarios posee serias falencias en los contenidos ligados a la práctica docente, contrariamente a la imagen pública actual que favorece a esta institución en desmedro de los Institutos Superiores de Formación Docente. Asimismo, los entrevistados valoran la formación académica y disciplinar recibida. Finalmente, sus relatos muestran la dificultad de las Universidades para auto-identificarse como formadoras de docentes para la escuela secundaria, así como la falta de acompañamiento por parte de los formadores en el espacio de las prácticas.

Palabras clave: ormaciónf docente - Profesores principiantes - Universidad - Escuela Secundaria Pedagogía.

\section{Abstract}

The following work stems from the outcomes of my Phd thesis in which I addressed the relationship between teacher training trajectories and employment insertion strategies of beginning high school teachers in Buenos Aires City.

Here I analyze the story of a group of interviewees from university, specifically from History, Geography and Literature course of studies, about their pedagogical formative experiences. Standing on the interpretive paradigm and a historical-cultural perspective, I analyze how the "two ways" of the Argentine teacher training performed particularities in the pedagogical trajectories of

* Doctora en Ciencias Sociales, Licenciada en Ciencias de la Educación, Licenciada en Historia, y Profesora de Nivel Medio y Superior en Historia por la Universidad de Buenos Aires (UBA). Actualmente se desempeña como Becaria Postdoctoral del CONICET con sede en el Instituto de Investigaciones en Ciencias de la Educación (IICE-FFyLUBA). Es formadora en Institutos Superiores de Formación Docente y profesora del área de Ciencias Sociales en escuelas secundarias de la Ciudad Autónoma de Buenos Aires. Su tesis de doctorado fue realizada en la Facultad de Ciencias Sociales de la UBA, bajo la dirección de Myriam Southwell. Fue financiada por una beca otorgada po el CONICET que dirigió Alejandra Birgin y co-dirigió Myriam Southwell y se desarrolló en el marco de dos Proyectos Ubacyt (Código: 20020130100051BA y 20020100100642BA) con sede en el IICE-UBA, ambos a cargo de Alejandra Birgin. 
REVISTA DE LA ESCUELA DE CIENCIAS DE LA EDUCACIÓN, AÑo 15, NRO. 14, VOL. 1, ENERO A JUNIO DE 2019. PÁGINAS 59-69. ISSN 1851-6297 (DESDE DICIEMBRE DE 2006 A DICIEMBRE DE 2017). ISSN 2362-3349 (EN LINNEA). DE RELATOS Y EXPERIENCIAS. ELEGIR LA UNIVERSIDAD PARA FORMARSE COMO PROFESOR DE LA ESCUELA SECUNDARIA. ANDREA IGLESIAS.

those who choose University to be trained as secondary school teachers.

The outcomes indicate that the pedagogical training received by the university students has serious shortcomings in content linked to teaching practice, contrary to the current public image that favors this institution at the expense of the specific teacher training institutions. Likewise, the interviewees value the academic and disciplinary training received. Finally, their stories show the difficulty that Universities have to identify themselves as teacher trainers for secondary school, as well as the lack of counseling of trainers during their initial practice experiences.

Keywords: Teacher training - Beginner teachers - University - Secondary School - Pedagogy.

\section{Introducción}

La formación docente en la Argentina se conformó desde sus inicios de manera fragmentada y heterogénea (Diker y Terigi, 1997) y con característica bifronte, ya que los profesores de nivel medio desde principios del siglo XX se formaban tanto en los Institutos de Formación Docente como en las Universidades. Esto implicó que ambos tipos de instituciones mantuvieran, desde el comienzo, contenidos y planes de estudio diferenciados (Birgin, 2014), características que persisten hasta la actualidad, con las complejidades que esto reviste para quienes optan por ser profesores de la escuela secundaria, como observaremos en los apartados siguientes. A partir del año 2006, con la sanción de la Ley de Educación Nacional ( $N^{\circ}$ 26.206) - LEN-y con la creación del Instituto Nacional de Formación Docente -INFoD-en el año 2007, los esfuerzos se centraron, precisamente, en políticas coordinadas con las Instituciones formadoras que permitieran reordenar el panorama nacional. La currícula contempló entonces tres campos de formación: el general (formación pedagógica), el específico (disciplinar) y la prácticas docentes (también denominadas "residencias"). Sin embargo, actualmente, en gran parte de las Universidades la diferenciación entre los planes de estudio de los profesorados universitarios y las licenciaturas se encuentra al finalizar la carrera, específicamente en el trayecto pedagógico (que varía en la cantidad de las materias según la institución).

En el caso de la Ciudad Autónoma de Buenos Aires -CABA-, donde radicó nuestra investigación, la Universidad de Buenos Aires ofrece para las carreras analizadas un ciclo pedagógico que consta de dos materias anuales: Didáctica General (basada en conocimientos generales de la Didáctica y la Pedagogía), y Didáctica Especial y Prácticas de la Enseñanza (orientada a la enseñanza específica de la disciplina, incluyendo las prácticas docentes), lo que implicó una valoración institucional menor para los títulos expedidos por esta Universidad respecto de los otorgados por los Institutos Superiores de Formación Docente -ISFD- (1). Dicha valoración responde a que se considera que la formación recibida en los ISFD es específica para el desarrollo de la tarea docente (2), mientras que la Universidad está asociada a la tarea de la investigación, aún para los egresados que optan por el trayecto pedagógico mencionado.

El presente trabajo se enmarca en mi tesis doctoral (3), donde analicé la relación entre la formación docente (inicial y continua) de los profesores principiantes y sus trayectorias laborales en las escuelas secundarias de gestión estatal y privadas de la CABA. Estos docentes se formaron tanto en Universidades como en ISFD y poseen trayectorias formativas y laborales diversas. Dada la característica bifronte de la formación docente, en nuestra investigación buscamos aportar a un elemento que consideramos relevante y que ha sido aún poco desarrollado en el campo pedagógico: el análisis conjunto de la formación de los docentes principiantes en las Universidades y en los ISFD. En el presente artículo nos centraremos específicamente en las experiencias de quienes eligieron la Universidad para formarse como profesores de escuela secundaria. Estimamos una contribución importante para el campo de la ciencia de la educación identificar las características del trayecto pedagógico dentro de la Universidad, así como la experiencia de sus protagonistas.

\section{Metodología}

Desde la perspectiva a la que abonamos, la teoría se desarrolla inductivamente en el propio proceso de investigación a través de la categorización que surge del análisis de los datos (Maxwell, 1996). Esta perspectiva orientó la propuesta metodológica y el trabajo de campo de la tesis doctoral mencionada, que consistió en el desarrollo de 24 entrevistas en profundidad (semi-estructuradas) a profesores principiantes, observación participante en espacios de socialización docente (cursos de formación docentes continua en instituciones estatales y sindicatos docentes, y Actos Públicos de distintas Juntas de Clasificación Docente de la CABA), así como el aporte de informantes claves, la exploración sobre las condiciones laborales, la recopilación de normativa e informes censales y de la oferta de formación de la Ciudad. El diseño metodológico de la investigación fue de tipo cualitativo y flexible (Mendizábal, 2006).

Se utilizó el método de comparación constante y un muestreo teórico, sin pretensión de representación estadística, seleccionando los casos que permitieran ampliar la teoría ya existente, mediante la saturación teórica. En paralelo a la recolección de datos, se desarrolló una etapa de codificación donde surgieron las categorías, que a partir de las similitudes y diferencias entre los casos, y a través de la comparación constante, se contrastaron con la teoría ya existente (Soneira, 2006).

El muestreo pretendió dar cuenta de la diversidad de trayectorias profesionales y perfiles docentes, por lo que la selección de los entrevistados en la tesis se realizó siguiendo el criterio de heterogeneidad a partir de las siguientes categorías: mujeres y varones, graduados y estudiantes de la formación inicial docente, formados en ISFD (de gestión estatal y gestión privada) y en Universidades estatales, que se 
REVISTA DE LA ESCUELA DE CIENCIAS DE LA EDUCACIÓN, AÑo 15, NRO. 14, VOL. 1, ENERO A JUNIO DE 2019. PÁGINAS 59-69. ISSN 1851-6297 (DESDE DICIEMBRE DE 2006 A DICIEMBRE DE 2017). ISSN 2362-3349 (EN LINNEA). DE RELATOS Y EXPERIENCIAS. ELEGIR LA UNIVERSIDAD PARA FORMARSE COMO PROFESOR DE LA ESCUELA SECUNDARIA. ANDREA IGLESIAS.

encuentren trabajando en escuelas secundaria de gestión estatal y de gestión privada de la CABA.

Los datos se integraron a través del ejercicio de la triangulación (Vasilachis de Gialdino, 1992) de las distintas técnicas mencionadas, lo que permitió lograr una mayor credibilidad de éstos (Maxwell, 1996), reduciendo así los sesgos propios de cada técnica de recolección. Consideramos que el análisis de los datos forma parte del diseño, por lo que éstese realizó de manera continua durante todo el proceso de investigación y no sólo al final de dicho proceso (Maxwell, 1996).

Dado que la investigación cualitativa genera una gran cantidad de material de registros, se realizó un análisis de los datos asistido por computadora a través del software Atlas.ti. Este software asistió en el proceso de la investigación para que puedan emerger las categorías de análisis desde la codificación, de manera de beneficiar y enriquecer el proceso de análisis (Chernobilsky, 2006). Asistidos por este software identificamos elementos significativos y recurrentes en el discurso de los entrevistados y en las observaciones realizadas, estableciéndose de este modo códigos y familia de códigos, así como conexiones entre los mismos, con el objetivo de generar relaciones de sentido que permitieron dar cuenta del fenómeno estudiado.

Sobre la muestra total de 24 entrevistas de la tesis, específicamente abordaremos en este artículo el conjunto de los 11 entrevistados (4) que eligieron Universidades estatales para formarse como profesores de escuela secundaria (en su mayoría en la Universidad de Buenos Aires), en las carreras de Historia, Geografía y Letras. La muestra analizada en este trabajo es heterogénea: 5 varones y 6 mujeres, con edades que se encuentran distribuidas por igual en dos rangos, 5 de los profesores entrevistados tienen entre 20 y 30 años, y 6 entre 30 y 40 años. Sus trayectorias son variadas. A excepción de una entrevistada, que constituye primera generación en su familia en acceder al nivel superior, el resto posee un núcleo familiar con educación superior. Una de las entrevistadas aún era estudiante y dos entrevistados no completaron el trayecto pedagógico y optaron directamente por la Licenciatura. En relación con sus biografías escolares, 4 asistieron a escuelas estatales, 3 a privadas y 3 combinaron ambas gestiones entre su escolaridad primaria y secundaria. Del mismo modo, 4 trabajan sólo en escuelas de gestión estatal, 3 en escuelas únicamente de gestión privada, y 4 trabajan en escuelas de ambos tipos de gestión.

Finalmente, estos profesores son denominados "principiantes" porque alcanzan hasta 5 años de trayectoria laboral dentro del sistema educativo (Menguini y Negrin, 2015). En nuestra investigación incluimos aquí distintos casos: en primer lugar, quienes ya obtuvieron su título docente; en segundo lugar, quienes poseen título habilitante porque optaron por la Licenciatura y no realizaron o no completaron el trayecto pedagógico en la Universidad; y en tercer lugar, quienes aún sin graduarse ya comenzaron a ejercer, ya que los definimos como profesores por su inserción laboral. Abordaremos a continuación los relatos de estos docentes sobre su formación en el ámbito de la Universidad.

\section{Experiencias formativas en la universidad}

Desde la literatura internacional se observa que los profesores principiantes critican fuertemente su formación inicial, respecto de la separación existente entre la teoría y la práctica, la presencia de contenidos abstractos, la falta de reflexión sobre la propia práctica y, fundamentalmente, la falta de relación de lo estudiado con el contexto de la inserción laboral (particularmente con las escuelas de sectores populares) (Périer, 2014; Marcelo, 2012; Villanueva Gutiérrez, 2010).

Desde el enfoque cualitativo adoptado en nuestra investigación, pretendemos comprender el significado que los sujetos le dan a la realidad, lo que a su vez le otorga sentido a sus acciones (Maxwell, 1996). En esa línea, analizaremos en este apartado cuál es la percepción que tienen los entrevistados que asistieron a la Universidad sobre su formación para la docencia.

Como mencionamos en trabajos anteriores (Miyazaki, 2017), los relatos de todos los profesores entrevistados dan cuenta de experiencias formativas distintivas entre las Universidades y los ISFD. En términos generales, la mayoría (tanto los formados en ISFD como en la Universidad) manifiestan que tuvieron una buena experiencia en su formación inicial y la consideran valiosa para enfrentar su tarea en el aula. Sin embargo, al consultarles específicamente sobre su formación para la docencia, encontramos distintas valoraciones.

En primer lugar, al igual que muestran investigaciones locales sobre las falencias en la formación pedagógica de los profesorados universitarios (De Amézola, 2011), los profesores que se formaron en esta institución coinciden en que su formación para la docencia fue insuficiente o nula. Como analizaremos luego, esta ausencia conforma una falencia importante de la Universidad en su rol formador para el nivel medio y superior.

En algunos casos, los entrevistados incluso abandonaron el trayecto pedagógico en la Universidad, producto del bajo nivel académico que encontraron, a diferencia de la licenciatura por la que finalmente optaron. A pesar de no poseer título habilitante para la docencia, observamos que en todos los casos logran insertarse laboralmente en las escuelas secundarias, en coincidencia con investigaciones que sostienen que en América Latina el acceso al puesto de trabajo bajo titulación no específica para la docencia va en aumento, en desmedro de su formación pedagógica, y en paralelo a la tendencia de la "credencialización del oficio" y la exigencia de profesionalización permanente. Del mismo modo, las investigaciones señalan que la masificación y obligatoriedad de la educación media implicó el aumento en la demanda de docentes para el nivel, lo que permitió tanto el ingreso de profesionales sin formación pedagógica como la inserción temprana de estudiantes de profesorado en las escuelas, flexibilizando los requisitos para acceder a este trabajo (Tenti Fanfani y Steinberg, 2011). 
REVISTA DE LA ESCUELA DE CIENCIAS DE LA EDUCACIÓN, AÑo 15, NRO. 14, VOL. 1, ENERO A JUNIO DE 2019. PÁGINAS 59-69. ISSN 1851-6297 (DESDE DICIEMBRE DE 2006 A DICIEMBRE DE 2017). ISSN 2362-3349 (EN LINNEA). DE RELATOS Y EXPERIENCIAS. ELEGIR LA UNIVERSIDAD PARA FORMARSE COMO PROFESOR DE LA ESCUELA SECUNDARIA. ANDREA IGLESIAS.

En segundo lugar, al consultarles por los contenidos del trayecto pedagógico, los entrevistados consideran que las materias cursadas no fueron de utilidad para la tarea en el aula. A veces, la valoración negativa viene asociada a los formadores y la calidad de sus clases, que ponen en duda. En otros casos, está asociada al contenido curricular de las materias. En definitiva, se considera que la formación fue escasa y se cuestiona el sentido y la utilidad de las materias pedagógicas:

La formación exclusivamente docente que tuve en la carrera [...] la sentí insuficiente. Insuficiente por lo siguiente; tenemos dos materias nosotros en Letras: Didáctica General y Didáctica Especial. Las dos anuales. Donde parece que lo único que tenés que hacer es ir. Con que vayas ya está [...] Cuando hice la especial también, era vení, cumplí, ya está [...] sinceramente fueron dos años que se pudieron haber aprovechado de otra manera. Cuando yo me tuve que parar en un aula estaba en pelotas (Entrevista Martina, 32, U, G, E, 3 años y 5 meses).

Según los entrevistados, su pasaje por la materia de Didáctica General no sólo no les aportó los contenidos generales esperados, sino que no observaron que tendiera puentes con el campo disciplinar que posteriormente abordarían desde la asignatura Didáctica Especial, como sería de esperar en este trayecto formativo (Devetac y Mastache, 2016). Asimismo, al final de este recorrido, los entrevistados cuestionan la "utilidad" de esta materia para su tarea docente:

Didáctica especial para la enseñanza de historia o sea... fue... a ver... cómo definirlo... poca, me parece que no, tampoco, ni totalmente útil [...] yo me imaginaba algo más práctico de darnos herramientas para trabajar en el aula con nuestra disciplina ... y terminamos gran parte de la materia, seguía siendo teoría acerca de la constitución del aula, de la gradualidad... interesante pero eso no me ayudaba a dar clases en el aula, saber del origen de la institución en la que estoy trabajando (Entrevista Sofía, 38, U, G, P, 2 años y medio).

Se repite en los entrevistados la idea de que estas materias que ellos esperaban que los formara para el ejercicio de la docencia, no les dieron las herramientas ni las respuestas frente a la incertidumbre que genera la inserción en el aula para un estudiante próximo a graduarse (Freitas y Nunes, 2017):

Muchas veces perjudica al propio discurso del saber. O sea, ¿cuál es el saber pedagógico? El saber pedagógico es "el encuentro"... Como si fuese una especie de lugar común que hay que repetir [...] Y la verdad que me dejaba en una incertidumbre bastante profunda (Entrevista Fabián, 32, U, G, E, 5 años).

Precisamente estas falencias en la formación docente inicial se observan más aún cuando los profesores se insertan en el aula por primera vez como principiantes. Aquí los entrevistados manifiestan que estas falencias les preocupan al enfrentarse con la tarea docente, en coincidencia con investigaciones de la región que sostienen que llegar al aula implica "el momento de mayor grado de fragilidad profesional" (Villanueva Gutiérrez, 2010, p. 263). En nuestra investigación recuperamos la categoría de "recién llegados" (Fernández Vavrik, 2016) para analizar cómo los docentes se insertan profesionalmente, realizando el "rito de pasaje" (Bourdieu, 2007) de "aspirantes" a profesores en ejercicio. Aún ajenos al trabajo de enseñar y desconociendo muchas veces las reglas del juego, buscan incorporarse al espacio áulico juntos a sus colegas. A los fines analíticos de la investigación, hemos denominado estas experiencias como "arribar al aula", lo que incluye una multiplicidad de relatos de los entrevistados en relación a sus primeras inserciones laborales en la escuela secundaria. Dado el objetivo de este artículo y la extensión del mismo, aquí sólo nos interesa retomar la categoría de "arribar" en relación con el contraste entre las primeras experiencias áulicas y la preparación recibida durante su formación inicial que mencionan los entrevistados:

Cómo se llena bien un libro de temas. Yo claro, yo lo llenaba intuitivamente por el casillero [...] Y ahí es donde digo, ¿pero esto no me lo tendrían que haber enseñado en la formación que tuve como docente de secundario en la facultad? Bueno, esa es una de las cosas que... O cómo armar bien un programa. O qué hacer cuando pasa algo dentro del aula que no sabés bien cómo controlar. O cómo articular algo tan estúpido como tu vida privada con los alumnos (Entrevista Fabián, 32, U, G, E, 5 años).

En tercer lugar, los entrevistados egresados de la Universidad realizan una diferenciación entre la formación recibida para ejercer la docencia en la escuela secundaria, y la del nivel superior, para el que claramente consideran que fueron mejor preparados. Podríamos decir también que esto es consecuencia de cómo es concebida la formación en la Universidad, más orientada a la investigación y menos a la docencia.

[la carrera] está muy perfilada en la Licenciatura. Es bueno para el que quiere hacer investigación. Pero para el que quiere dedicarse a la docencia es malo. Es como que ahí hay un punto de tensión. Porque todas las materias están dadas a veces en un nivel de complejidad que no usas para enseñar en la secundaria. Que no te sirve el contenido para enseñar en la secundaria. Y porque me faltó Historia fáctica. Mucha Historia fáctica. (Entrevista María, 22, U, G, E y P, 7 meses)

Como cuarto elemento, encontramos en el relato de los entrevistados la inserción profesional temprana como compensatoria de la falta de formación para el aula, es decir, que la experiencia y la 
REVISTA DE LA ESCUELA DE CIENCIAS DE LA EDUCACIÓN, AÑo 15, NRO. 14, VOL. 1, ENERO A JUNIO DE 2019. PÁGINAS 59-69. ISSN 1851-6297 (DESDE DICIEMBRE DE 2006 A DICIEMBRE DE 2017). ISSN 2362-3349 (EN LINNEA). DE RELATOS Y EXPERIENCIAS. ELEGIR LA UNIVERSIDAD PARA FORMARSE COMO PROFESOR DE LA ESCUELA SECUNDARIA. ANDREA IGLESIAS.

práctica les permiten desarrollar habilidades pedagógicas (Tenti Fanfani, 2005). De este modo, el consejo de sus colegas en la escuela, así como vivenciar el aula, los ayudaron a compensar esa falencia percibida en la formación inicial, confirmando la tesis de que "aprender en la práctica es un modo de socialización laboral en el que las instituciones juegan un papel fundamental" (Mereshián y Calatayud, 2009, p. 259). Elemento este último que toma aún más relevancia para el caso de los profesores que arriban al aula.

Investigaciones realizadas en el ámbito nacional también muestran que los futuros profesores consideran que es en la práctica como se aprende a enseñar, junto a los colegas más experimentados, supliendo las "vacancias" de la formación docente inicial, y "desenganchando" de este modo al saber pedagógico del saber disciplinar, sobre el que suelen tener una valoración más alta (Terigi, 2011), más aún los que se formaron en la Universidad. Tanto el rol de la práctica áulica para subsanar las falencias sobre el saber pedagógico, así como la mayor ponderación del saber disciplinar, y el aprendizaje junto a los colegas, son todos elementos que están presentes en el relato de nuestros entrevistados egresados de la Universidad.

Para finalizar, debemos hacer aquí una excepción con los profesores de Geografía, ya que en la Universidad estos profesores tienen el trayecto del profesorado más largo (6 materias, en lugar de las 2 materias que poseen las carreras de Letras e Historia) (5). En este sentido, estos profesores plantean en las entrevistas que su formación pedagógico-didáctica los preparó para la práctica áulica, valorando su formación para la docencia, diferenciándose claramente del resto de sus colegas de Ciencias Sociales de la misma Universidad:

Y después al año siguiente hice la Didáctica Especial de [Geografía], y el seminario este que te contaba. Y claro, ahí ya estar en algo más referido a Geografía era otra cosa. Pero ahí también me di cuenta que un montón de cuestiones que había visto en Didáctica I, Didáctica II, me había servido para pensar la enseñanza [...] Para mí la formación docente es buena. Por más de que uno reniegue, las padezca, diga “¿por qué no me tocó Didáctica General?”, para mí está bueno que tengamos Didáctica I y Didáctica II [...] La planificación anual, la planificación diaria, todas esas cosas que te suenan re lejanas cuando las cursás, pero después lo tenés que hacer. Cuando entrás a trabajar a una escuela te piden: "bueno, mirá $1^{\circ}$ año cambió el plan de estudios, tenés que hacerlo de nuevo". Como esa cuestiones, y me parece que eso sí es valioso. (Entrevista Catalina, 27, U, E, P, 4 años).

Precisamente aquí observamos cómo para el caso de los profesores de Geografía la formación sí les brindó herramientas necesarias para la práctica docente. Precisamente, diversos autores del campo pedagógico resaltan que uno de los problemas de la formación es la compleja relación teoría-práctica y la lejanía entre la preparación recibida y el ejercicio docente (Diker y Terigi, 1997), así como la falta de capacitación para enseñar a los "alumnos reales" de las nuevas generaciones que se incorporaron a la escuela (Rayou \& Ria, 2009).

\section{Debates y tensiones sobre el sistema de formación docente \\ 4.1. Elegir la universidad}

En nuestra investigación, a los fines analíticos, agrupamos las razones por las que los entrevistados eligieron la institución formadora a la que asistieron, en el contexto de la complejidad que reviste la formación docente en nuestro país. Como mencionamos en el apartado metodológico, mediante el ejercicio de la triangulación de los datos obtenidos del campo de la investigación y ante las recurrencias en las respuestas de los entrevistados, construimos tres dimensiones de análisis para esta elección: una primera institucional, otra relacionada con los vínculos personales, y una tercera vinculada a la proyección profesional.

Comenzando con la dimensión institucional, observamos en la elección de la institución formadora motivos tales como: el tipo de acompañamiento a los alumnos, el tipo de formación buscada, la comodidad o cercanía (con el hogar y/o el lugar de trabajo). Esta última se corresponde particularmente con los casos en los que se observa un desconocimiento de las opciones disponibles, al igual que muestran otras investigaciones (Charovsky, 2013). Asimismo, se destaca la justificación por el prestigio de la institución formadora, con mayor fuerza en el caso de los que optaron por la Universidad.

La [Universidad] cuando salís del secundario es casi la opción que tenés, la opción básica que tenés. Es el lugar donde todo el mundo te dice que tenés que estudiar cuando sos chico, que es el mejor lugar. Y así uno va como directamente [...] me anoté y empecé a estudiar (Entrevista Lara, 35, I, G, E, 3 años).

Creo que para la mayoría de los que vivimos en la Ciudad [...] Es una Universidad de prestigio, gratuita, pública. A menos que tengas una excelente referencia de otra Universidad, parece natural ir por ese camino (Entrevista Ariel, 26, U, G, E, 1 año).

En segundo lugar, muchos profesores eligieron en base a vínculos personales, recomendaciones de sus profesores de la escuela secundaria, o por el consejo de familiares que asistieron a estas instituciones respondiendo a lo que llaman una "tradición familiar". Particularmente, la recomendación de los profesores en la escuela secundaria se encuentra más presente entre quienes optaron por los ISFD que para quienes eligieron la Universidad, orientación valorada frente a la desinformación que manifestaron tener al momento de la elección. 
REVISTA DE LA ESCUELA DE CIENCIAS DE LA EDUCACIÓN, AÑo 15, NRO. 14, VOL. 1, ENERO A JUNIO DE 2019. PÁGINAS 59-69. ISSN 1851-6297 (DESDE DICIEMBRE DE 2006 A DICIEMBRE DE 2017). ISSN 2362-3349 (EN LINNEA). DE RELATOS Y EXPERIENCIAS. ELEGIR LA UNIVERSIDAD PARA FORMARSE COMO PROFESOR DE LA ESCUELA SECUNDARIA. ANDREA IGLESIAS.

Finalmente, aunque con menor recurrencia entre los entrevistados, aparece la elección de la institución en base a la proyección profesional. Este último punto está vinculado con lo que analizaremos en el siguiente apartado, ya que muchos entrevistados que sabían que querían dedicarse a la docencia eligieron los ISFD, dada la formación específica para la profesión que esperaban recibir. En cambio, quienes eligieron la Universidad, lo hicieron esperando mayores y variadas probabilidades de inserción laboral:

Y ahí es donde hablamos que el título de la Facultad... digamos que tiene una salida importante en cuanto a las materias en cuanto lo que uno puede dar [...] al haber estudiado en la Facultad hay como una amplitud de disciplinas que maneja y que puede rendir (Entrevista Sofía, 38, U, G, P, 2 años y medio).

El relato de los entrevistados coincide con estudios que relevan la opinión de estudiantes de formación docente en el país, donde la elección por la institución formadora aparece relacionada a la recomendación de familiares y de profesores, a la cercanía geográfica y al prestigio de la institución, como en el caso de la CABA por la variedad en las opciones formativas (Tenti Fanfani, 2010). Asimismo, las distintas opciones que toman los futuros profesores, se relacionan también con otros elementos, por fuera de las condiciones objetivas y los mundos posibles, pero que igualmente delimitan las trayectorias docentes, a saber: sus expectativas, deseos, frustraciones y representaciones individuales acerca de las posibilidades y limitaciones dentro del campo profesional, es decir, a través de lo que Dubar (1994) llama una "trayectoria subjetiva", por la cual los sujetos vinculan la reconstitución de su pasado y la proyección en el futuro.

\subsection{Visiones contrapuestas: el Instituto o la Universidad}

Retomando el debate existente en América Latina, en las últimas décadas se viene discutiendo el rol de la Universidad en la formación docente y el pasaje de la formación inicial hacia estas instituciones, así como la falta de preparación pedagógica en el caso de los profesionales que se incorporan al cuerpo docente (6). Algunos autores encuentran que la formación docente en la región se perfila hacia "un irreversible proceso de universitarización", producto de ciertos cambios recientes, como ser:

Los procesos de articulación terciario-universitario, la creación de los ciclos de licenciatura, la posibilidad de la conversión de institutos terciarios en colegios universitarios, y las propias demandas profesionales, todo ello conlleva, con distintos órdenes de influencia a que -sin saber cuándo- la formación docente se transforme y pase a ser un producto típicamente universitario (Cámpoli, 2004, p. 106).

Este debate está íntimamente relacionado con la denominada "calidad" de la formación de los futuros docentes. Existe cierto consenso desde la opinión pública acerca de que la Universidad puede ofrecer mejor formación que otras instituciones de nivel superior o nivel terciario. Este debate recrudeció a la luz de un proyecto de ley, recientemente aprobado, que envió el Ejecutivo de la CABA a la Legislatura porteña, creando la "UniCABA" (Universidad de Maestros de la Ciudad Autónoma de Buenos Aires). Esta institución reemplazaría a los 29 ISFD que tiene actualmente la Ciudad, por lo que generó una gran resistencia de la comunidad educativa y académica desde su anuncio por la prensa a fines del año 2017 (7) (Miyazaki, 2018).

En Argentina, la heterogeneidad y fragmentación del sistema formador, junto a la diferenciación de los contenidos y planes de estudio, ha complejizado históricamente la articulación de las instituciones. En este sentido, la opinión de los especialistas en general apuesta a que "una política para la educación superior deberá dotar de mayor integración e institucionalidad al sistema formador, superando la fragmentación, la atomización y la primarización que lo ha caracterizado" (Piovani, 2013, p. 225).

Estas discusiones están presentes en las propias instituciones y en el testimonio de los profesores entrevistados. Históricamente, producto de la conformación bifronte, se consideró que la Universidad y los ISFD tenían distintos tipos de formación y de públicos. Esa conformación histórica fue cambiando en las últimas décadas (Carli, 2012; Rinesi, 2015). Sin embargo, pareciera ser que ciertas ideas tomaron carácter de verdad. En el caso de los profesores universitarios, la comparación entre instituciones implica una fuerte crítica a la formación docente recibida, en relación con lo que ellos consideran reciben sus colegas de los ISFD:

La ventaja, creo, que tienen la mayoría de los Institutos de Profesorado, a diferencia del profesorado universitario, es que tiene más ese manejo con los adolescentes, con los alumnos. Porque hasta donde sé, ellos las prácticas las tienen más seguido y antes. Nosotros tuvimos prácticas solamente. O sea, yo me recibí con prácticas. Al final de la carrera. Vos te hacés toda la carrera y después vas a la práctica. Si no te gusta te hiciste toda la carrera al pedo. Fuiste a dar clases y no te gustó, hiciste la carrera al pedo (Entrevista María, 22, U, G, E y P, 7 meses).

Esta visión coincide con la que tienen los graduados de ISFD, ya que también ellos marcan la diferencia en el contenido pedagógico aprendido, ponderando el saber específico para el ejercicio de la docencia, y la distancia que encuentran con la Universidad que forma para la investigación.

Por su parte, los graduados universitarios observan una falta de contenido disciplinar en los ISFD que, en muchos casos, perciben en sus colegas de la escuela: 
REVISTA DE LA ESCUELA DE CIENCIAS DE LA EDUCACIÓN, AÑo 15, NRO. 14, VOL. 1, ENERO A JUNIO DE 2019. PÁGINAS 59-69. ISSN 1851-6297 (DESDE DICIEMBRE DE 2006 A DICIEMBRE DE 2017). ISSN 2362-3349 (EN LINNEA). DE RELATOS Y EXPERIENCIAS. ELEGIR LA UNIVERSIDAD PARA FORMARSE COMO PROFESOR DE LA ESCUELA SECUNDARIA. ANDREA IGLESIAS.

Nunca fui a un profesorado, nunca cursé en un profesorado, pero sí revisé programas, conocí gente que fue a profesorados. Y siempre lo que muestra una currícula más o menos estándar, ves un montón de materias de didáctica, y muy pocas referidas a conocimientos específicos [...] creo que un profesor formado en la [Universidad] tiene muchísimo (enfatiza) más conocimiento que cualquier profesor del profesorado. Porque lamentablemente los profesorados están tan articulados con este problema del discurso didáctico, que tienen un montón de materias de didáctica [...] Y después cuando les preguntás de Literatura no tienen ni idea (enfatiza). Entonces es un problema, no tienen una relación amorosa con el objeto, que para mí es fundamental para lo que es el ejercicio de la clase, sobre todo a nivel secundario (Entrevista Fabián, 32, U, G, E, 5 años).

Si bien todos los graduados de la Universidad acuerdan en la distancia que existe entre la formación para la disciplina recibida en su institución y en un ISFD, también en algunos casos manifiestan que hay matices:

Yo creo que hay mucho prejuicio de decir que en los Profesorados no hay buena formación en cuanto al tema de conocimiento [...] la parte didáctica incluso para los estudiantes es muy pobre. Como que no está reconocido como algo necesario [en la Universidad]. En cambio en el Profesorado todo gira en torno a eso. Entonces me parece que en cuanto a formación docente es mejor. Pero tienen que estar siempre las dos patas también, en cuanto al conocimiento. (Entrevista Valeria, 28, U, G, P, 1 año y medio).

Los profesores entrevistados coinciden en que esta preparación en los contenidos pedagógicos y didácticos implica necesariamente mejor formación para el aula. Los egresados de la Universidad lo observan en su práctica como profesores y lo destacan en las entrevistas:

Veo que los profesorados tienen más esta pata pedagógica, más de la institución, con más comprender la escuela, o comprender a los sujetos, que son parte de la escuela y que nosotros no lo tenemos [...] Estamos muy preparados geográficamente digamos, pero no en lo que es la pedagogía (Entrevista Catalina, 27, U, E, P, 4 años).

La diferencia radica para otros entrevistados en que los ISFD forman para la docencia. A su vez, esta especificidad se relaciona con las razones de la elecci ción formadoraón de la institua partir de la imagen que cada entrevistado tenía al elegir su carrera. Los ISFD son identificados claramente por los entrevistados como los formadores de docentes, y la Universidad es vista únicamente por su función de formar licenciados e investigadores. Esto se evidenció en las entrevistas realizadas a los profesores graduados de ISFD que, muchas veces, sólo asociaban a las Universidades con las licenciaturas, pero no las reconocían como instituciones formadoras de docentes para el nivel medio y superior. De hecho, esta es la razón por la que manifestaron que no las tuvieron en cuenta para formarse como docentes y se enteraron luego de que existía la opción de los profesorados universitarios.

Además de la falta de formación pedagógica que mencionan los entrevistados, encontramos que en las materias específicas de formación docente dentro de la Universidad, la enseñanza en la escuela secundaria como salida laboral es desprestigiada por los propios formadores:

Sentía que ya desde el vamos nos estaban repeliendo. Las profesoras que daban Didáctica en ese momento venían y te decían "yo sé que todos ustedes están acá porque se quieren recibir temprano y no quieren hacer una tesis". Yo pensaba "¿pero esta mina qué me está diciendo?, ¿qué sabe si yo quiero ser docente? Si yo entré acá para ser docente." [...] Entonces sentí todo el tiempo que nos estaban combatiendo, cuando en realidad estaban desperdiciando un espacio que podrían usar para invitarnos a ser docentes [...] el rechazo de la facultad hacia la docencia. ¿Por qué desde el vamos nos quieren decir que no? ¿Y por qué cuando vamos a adquirir herramientas para hacerlo nos dicen que no queremos estar ahí? ¿Por qué se sobrevalora la docencia universitaria y se le tira porquería a la docencia media, cuando en realidad ahí es donde más tenés que laburar? (Entrevista Martina, 32, U, G, E, 3 años y 5 meses).

Como pudimos observar, los graduados de la Universidad son muy críticos de su falta de formación pedagógica. Sin embargo, reivindican la alta calidad de la formación académica que recibieron, por la que afirman que volverían a elegir la Universidad para formarse. Podríamos decir, a modo de hipótesis, que a pesar de reconocer las falencias en su formación docente inicial, estos profesores le otorgan más valor formativo al conocimiento disciplinar, restándole así importancia a los saberes pedagógico-didácticos necesarios para el ejercicio de la profesión.

En la voz de los profesores entrevistados vemos reflejada aquella concepción de la "tradición academicista" de la Universidad que prioriza la formación en la disciplina, en desmedro de la formación pedagógica, a la que adjudican "falta de rigor científico, describiéndola como superficial e innecesaria" (Molinari, 2005, p. 28). Esta es una explicación posible de la fortaleza en la preparación académica que resaltan quienes se formaron en la Universidad, en coincidencia con lo que manifiestan sobre sus colegas formados en los ISFD. A su vez, esta escisión teoría-práctica, que mencionan reiteradamente los graduados de la Universidad, muestra en general una "relación de exterioridad" con el saber didáctico (Muñoz, 2016).

Estas tensiones presentes entre las dos tradiciones formativas de los ISFD y Universidad, como ya 
REVISTA DE LA ESCUELA DE CIENCIAS DE LA EDUCACIÓN, AÑo 15, NRO. 14, VOL. 1, ENERO A JUNIO DE 2019. PÁGINAS 59-69. ISSN 1851-6297 (DESDE DICIEMBRE DE 2006 A DICIEMBRE DE 2017). ISSN 2362-3349 (EN LINNEA). DE RELATOS Y EXPERIENCIAS. ELEGIR LA UNIVERSIDAD PARA FORMARSE COMO PROFESOR DE LA ESCUELA SECUNDARIA. ANDREA IGLESIAS.

mencionamos, remiten a un debate más amplio en América Latina sobre la formación de los futuros docentes. Como hemos analizado en trabajos anteriores, existe en la opinión pública un consenso acerca de que la Universidad ofrece una formación de mejor calidad que los Institutos, por lo que sería deseable que todos los docentes se formaran allí (Miyazaki, 2015).

Sin embargo, distintas investigaciones de la región y locales muestran que la exportación del modelo universitario al resto del sistema de educación superior no garantiza la formación de "mejores" docentes ni resuelve la lejanía de la preparación de las aulas "reales" en las que se insertan (Diker y Terigi, 1997). La palabra de los profesores sobre la propia institución que los formó pone en tensión esa imagen construida por los medios masivos y la opinión pública, resignificando estos discursos sobre la noción de docente (Southwell y Vassiliades, 2014), y reapropiándose del sentido de su formación. No obstante, podríamos afirmar, a modo de hipótesis, que esta construcción que hace la prensa coincide en algunos testimonios con el perfil formativo que los profesores observan de sus colegas, por lo que la tensión no se resuelve, del mismo modo que los sentidos no son fijos y se encuentran en constante negociación.

Analizar las valoraciones de los profesores formados, en una u otra institución, nos ayuda a desentrañar este tipo de discurso que pretende absolutizar y cristalizar en cada una de ellas un tipo de formación que, en general, termina operando a favor de la Universidad y en desmedro de los ISFD. Por el contrario, a través de otros relatos de los entrevistados de ambas instituciones se observan distintos elementos de la formación, sin que ninguna de ellas le garantice por sí misma transformarlo en el "mejor" docente al llegar al aula:

[Quienes se formaron en la Universidad] en comparación con quienes son docentes de terciaria digamos, tienen mucha más formación. No necesariamente eso significa que vayan a ser excelentes docentes (se ríe), pero bueno, tienen más formación pedagógica, mucha más (...) Muchas veces no la aplican, pero la tienen. (Entrevista Mercedes, 33, U, G, E y P, 4 años y medio).

Finalmente, otro elemento para resaltar es la diferenciación que se establece entre ambas instituciones en relación con el recibimiento y acompañamiento de los alumnos. Sobre todo para aquellos que transitaron ambas instituciones, rescatan el valor del seguimiento al alumno y un trato más "humano" en los ISFD, generando así un sentimiento de "pertenencia" que los diferencia de las Universidades.

Estaba muy enojada [con la Universidad]. Era demasiado, demasiado sacrificio que no tenía que ver con lo académico, con lo intelectual, sino que tenía que ver con otras cuestiones que no soportaba. Y [con la Universidad] me quedó mucho resentimiento [...] En [la Universidad] vos, claro, es un ambiente muy universitario en donde no tenés un gran contacto con los profesores. Con los docentes. Vos siempre pasás como un número, una cosa que está ahí sentada, escucha, y de vez en cuando entregás un parcial. En el [ISFD] hay otro tipo de relación, tanto con los compañeros como con los docentes. Primero porque los tenés más tiempo. Y segundo porque como está planteado como un espacio de enseñanza pedagógica, eso intenta también reproducirse dentro de las aulas. Entonces la relación suele ser más cercana y vos tenés otro tipo de trato. Realmente sentís una pertenencia más fuerte que la que podés sentir en la [Universidad] [...] Así que por ese lado el [ISFD] te hace sentir parte de la institución. Y eso creo que fue positivo (Entrevista Lara, 35, I, G, E, 3 años)

Se resalta aquí la identificación que generaran los ISFD con sus estudiantes así como el rol de los formadores, el acompañamiento recibido, la formación "humana" que los entrevistados subrayan. Como muestran otras investigaciones locales (Charovsky, 2013), para quienes transitaron por ambas instituciones, frente a la "orfandad" que sintieron en la Universidad, el ISFD aparece como un lugar capaz de alojar y recibir a sus estudiantes. Es allí donde radica ese sentido de la "hospitalidad", inexistente en la Universidad según los relatos:

[En el ISFD] estás todo el tiempo acompañado. Compartís experiencias de cursada, material. Es muy familiar en ese sentido. Por lo tanto se me hizo mucho más fácil [con respecto a su experiencia previa en la Universidad], en ese sentido de acompañamiento, de conocer a alguien, y de poder hablar con alguien (Entrevista Gabriel, 34, I, G, P, 2 años)

Entendemos que el sistema de cursada de los ISFD, a diferencia del de la Universidad, puede a su vez facilitar esta cercanía con los alumnos. Sentirse acompañados durante su período formativo es recurrentemente mencionado por los graduados de los ISFD, y ponderado como uno de los rasgos sobresalientes de su formación:

Lo que me gustó mucho siempre [del ISFD] fue esta cuestión del ambiente. A mí me gusta mucho sentirme cómodo donde estoy estudiando, donde estoy trabajando. Y esto me gustó mucho. [...] Por ahí parece bastante infantil el pensamiento, pero es como que sentir compañía y que te estén preguntando "che, cómo te fue el otro día con tal cosa", y que se acuerden que estuvieron hablando con vos en el colectivo está bueno. Uno se siente bien (Entrevista lan, 23, I, E, P, 6 meses).

Como pudimos observar, cada institución posee características específicas, pero también compartidas. EI ISFD es un espacio que se habita, se ocupa, genera una identificación y es donde los alumnos tienen una identidad propia. La Universidad se vive y se transita, es un lugar de paso para 
REVISTA DE LA ESCUELA DE CIENCIAS DE LA EDUCACIÓN, AÑo 15, NRO. 14, VOL. 1, ENERO A JUNIO DE 2019. PÁGINAS 59-69. ISSN 1851-6297 (DESDE DICIEMBRE DE 2006 A DICIEMBRE DE 2017). ISSN 2362-3349 (EN LINNEA). DE RELATOS Y EXPERIENCIAS. ELEGIR LA UNIVERSIDAD PARA FORMARSE COMO PROFESOR DE LA ESCUELA SECUNDARIA. ANDREA IGLESIAS.

algunos y de permanencia para otros, que de igual forma deja marca en quien la recorre, la huella de vivir la experiencia universitaria (Carli, 2012), una experiencia que al transitarla transforma al sujeto (Larrosa, 2003). Estas trayectorias formativas dejan entrever a su vez la inexistencia de un cuerpo homogéneo de docentes (Arroyo y Poliak, 2008; Southwell y Vassiliades, 2014), donde la conformación bifronte de la formación dibuja a priori trayectorias formativas distintas.

\section{Reflexiones finales}

Hemos observado a lo largo del presente trabajo que los profesores entrevistados que eligieron la Universidad para formarse distinguen entre lo que se considera la formación pedagógica, un saber específico para el trabajo en el aula, y la formación académica, relacionada con el estudio de la disciplina (Muñoz, 2016). Del mismo modo, observamos que los profesores naturalizan en sus relatos la escisión teoría-práctica, ya que el conocimiento académico se presenta como un "pensamiento privilegiado", y el saber proveniente de la práctica en el aula aparece como un conocimiento de "segunda categoría" (Davini, 2015). Encontramos en sus discursos una dicotomía teoría/práctica, saber/hacer, en la que el docente "aplica" o "baja" al aula los conocimientos aprendidos durante la formación (Alliaud, 2017). Los profesores de Geografía se conforman como una excepción, aunque parcial, a este posicionamiento, puesto que, aunque tuvieron mayor cantidad de materias pedagógicas y consideran que su formación para la docencia fue mejor que la de sus colegas de la misma Universidad, también sostienen en sus relatos esta dicotomía.

La pedagogía está ausente o aparece desdibujada en la formación docente inicial recibida en la Universidad, lo que complejiza aún más el ingreso al aula para los profesores principiantes que, en algunos casos, es compensada parcialmente por la inserción temprana en el aula antes de recibirse. En vínculo con la conformación bifronte del sistema formador y la elección de esta institución formadora, la formación académica y la formación disciplinar en la Universidad (Muñoz, 2016) protagonizan los relatos, desdibujando así el "potencial formativo" de la formación pedagógica (Davini, 2015).

En este sentido, los graduados de la Universidad ponderan el alto nivel de la formación académica y disciplinar recibidas distanciándose de los graduados de los ISFD. A primera vista, esto coincide con la imagen pública negativa a cerca de las falencias en la preparación que brindan estas instituciones, mostrando como deseable que la formación docente se realice en la Universidad (Miyazaki, 2015), como ocurre en otros países de la región. En contraposición, observamos cómo otro grupo de entrevistados se separa de esta visión al remarcar las falencias que la Universidad tiene en la formación específica en el ejercicio de la docencia.

Podemos concluir entonces que el testimonio de algunos entrevistados relativiza ese discurso público, construido con carácter de verdad desde los medios masivos de comunicación, actores centrales que conforma la opinión pública y la agenda política (Chiroleu, 2012). Si bien ésteno fue el objetivo de nuestra indagación, constituye una vacancia y sería un aporte al campo de la pedagogía y la formación docente poder repensar en futuras líneas de investigación qué relación existe entre la construcción de ese discurso y la percepción que tienen los propios docentes sobre su formación.

A modo de cierre, podríamos decir que la crítica de los entrevistados que eligieron la Universidad para formarse como profesores reedita la discusión histórica sobre la importancia del saber específico pedagógico de la práctica docente, su valor frente al saber disciplinar, así como los orígenes de la docencia para la escuela secundaria y la tensión entre la autonomía universitaria y la regulación nacional (Birgin, 2014). Asimismo, la experiencia formativa de los entrevistados muestra la dificultad de las Universidades en auto-reconocerse como formadoras de profesores para la escuela secundaria, como parte de la Educación Superior que es responsable de esta tarea. Se abren así nuevos interrogantes sobre la vinculación que tienen las Universidades con el saber pedagógico, por un lado, y los ISFD con el saber disciplinar, por otro. Al mismo tiempo, en futuras investigaciones desearíamos profundizar sobre las maneras en que las políticas públicas pueden fortalecer la articulación entre ambos subsistemas de la formación docente, con larga tradición en la Argentina, y frente a la embestida pública que sufre actualmente la docencia como profesión.

\section{Notas bibliográficas}

(1) Según el relevamiento realizado, distintas Juntas de Clasificación Docente otorgan al mismo título diferentes puntajes, sin existir una razón aparente o explicitada en la normativa que lo justifique. El título de Profesor de Nivel Medio y Superior otorgado por la Universidad de Buenos Aires posee en algunos casos 3 puntos menos (título habilitante no docente, 6 puntos) que los otorgados por los ISFD (título docente, 9 puntos) (Estatuto Docente GCBA, Art. 15).

(2) Las materias relacionadas con la formación docente varían según el ISFD entre 10 y 16 para planes de estudio que poseen entre 40 y 50 asignaturas.

(3) Tesis titulada "Trayectorias de formación y estrategias de inserción laboral. Los profesores principiantes de las escuelas secundarias de la Ciudad Autónoma de Buenos Aires" (FSoC, UBA), Directora Myriam Southwell. Financiada por una beca doctoral CONICET dirigida por Alejandra Birgin y co-dirigida por Myriam Southwell y en el marco de un Proyecto Ubacyt (Código: 20020130100051BA) a cargo de Alejandra Birgin, ambos con sede en el Instituto de Investigaciones en Ciencias de la Educación de la Universidad de Buenos Aires (IICE/UBA).

(4) Sin desconocer el debate actual al respecto, decidimos utilizar aquí el masculino gramatical para referirnos al colectivo mixto, incluyendo a los y las profesores y profesoras principiantes. Asimismo, para preservar la 
REVISTA DE LA ESCUELA DE CIENCIAS DE LA EDUCACIÓN, AÑo 15, NRO. 14, VOL. 1, ENERO A JUNIO DE 2019. PÁGINAS 59-69. ISSN 1851-6297 (DESDE DICIEMBRE DE 2006 A DICIEMBRE DE 2017). ISSN 2362-3349 (EN LINNEA). DE RELATOS Y EXPERIENCIAS. ELEGIR LA UNIVERSIDAD PARA FORMARSE COMO PROFESOR DE LA ESCUELA SECUNDARIA. ANDREA IGLESIAS.

confidencialidad y la identidad, se modificaron sus nombres y los datos de las instituciones de formación y las escuelas mencionadas en las entrevistas. Los fragmentos citados se referenciaron del siguiente modo: alias, edad, institución formadora -ISFD (I) o Universidad (U)-, estudiante (E) o graduado (G); tipo de gestión de escuelas en las que trabaja -estatal (E) o privada (P) - antigüedad en la docencia (años/meses).

(5) En lugar de cursar Didáctica General y Especial, los estudiantes de Geografía cursan Didáctica I y II, dos materias específicas que dicta la carrera de Ciencias de la Educación de la misma Facultad, junto con otras cuatro materias pedagógicas ligadas a la práctica áulica en la escuela secundaria.

(6) Del mismo modo, existe una preocupación en la región, cristalizada en los informes de los Organismos Internacionales, por el proceso de "acreditación" de todas las instituciones formadoras en cada país; mejorar el acceso a la carrera; "brindar apoyo a los profesores principiantes"; favorecer la "formación en ejercicio" y el desarrollo profesional de los docentes (OEI, 2010, p. 254-255)

(7) Para mayor información sobre el proyecto véase http://www.buenosaires.gob.ar/noticias/documentos-detrabajo-sobre-la-creacion-de-la-unicaba-0 [consultado 30/10/2018].

\section{Referencias bibliográficas}

- $\quad$ Alliaud, A. (2017). Los artesanos de la enseñanza. Acerca de la formación de maestros con oficio. Paidós, Buenos Aires.

- $\quad$ Arroyo, M. y Poliak, N. (2008). "Discusiones en torno a fragmentación, identidades y compromiso. Enseñar en las Escuelas de Reingreso". En: Tiramonti, G. (Dir.) Variaciones sobre la forma escolar. Límites y posibilidades de la escuela media, 89-124. Flacso/Homo Sapiens, Rosario. Recuperadohttp://www.amsafe.org.ar/formacion/images/2012ConcursoNormales/Variacionessobrelaformaescolar.pdf [consultado 30/10/2018]

- Birgin, A. (coord.) (2014). Estudio sobre criterios de calidad y mejora de la formación docente del MERCOSUR. Teseo/OEI/ Programa de Apoyo al Sector Educativo del MERCOSUR (PASEM)/MERCOSUR, Buenos Aires.

- $\quad$ Bourdieu, P. (2007). El sentido práctico. Siglo XXI, Buenos Aires.

- Cámpoli, O. (consultor) (2004). La formación docente en la República Argentina (Documento de trabajo). IESALC/IES/UNESCO, Buenos Aires. Recuperado http://www.oei.es/docentes/info_pais/informe_formacion_docente_argentina_iesalc.pdf [consultado 30/10/2018].

- Carli, S. (2012). El estudiante universitario. Hacia una historia de la educación pública. Siglo XXI, Buenos Aires.

- Charovsky, M. (2013). La fragmentación de la formación docente. Su relación con el sistema educativo. Estudio de caso en el partido de Pilar, provincia de Buenos Aires. Tesis de Maestría. Facultad de Filosofía y Letras. Universidad de Buenos Aires. Buenos Aires [MIMEO].

- Chernobilsky, L. (2006). "El uso de la computadora como auxiliar en el análisis de datos cualitativos". En: Vasilachis de Gialdino, I. (coord.) Estrategias de investigación cualitativa. pp. 239-273. Gedisa, Barcelona.

- Chiroleu, A. (2012). "La política universitaria como política pública". En: Chiroleu, A., Suasnábar, C. y Rovelli, L. Política universitaria en la Argentina: revisando viejos legados en busca de nuevos horizontes. pp 13-31. Universidad Nacional de General Sarmiento/Instituto de Estudios y Capacitación (IEC) Federación Nacional de Docentes Universitarios, Buenos Aires.

- Davini, M. C. (2015). La formación en la práctica docente. Paidós, Buenos Aires.

- De Amézola, G. (2011). "La formación del profesor de historia en la Universidad Nacional de La Plata". En: Clio \& Asociados. (15), pp. 178-195. http://www.clio.fahce.unlp.edu.ar/article/view/clion15a11/5154 [consultado 30/10/2018].

- Devetac, R y Mastache, A. (2016). "Aportes desde la Didáctica General a la Didáctica Específica en el marco de la enseñanza de las Ciencias Jurídicas". En Insaurralde, M. (Coord.) Enseñar en las Universidades y en los Institutos de Formación Docente (pp. 113-120). Noveduc, Buenos Aires.

- $\quad$ Diker, G. y Terigi, F. (1997). La formación de maestros y profesores: hoja de ruta. Paidós, Buenos Aires.

- Dubar, C. (1994). L'insertion comme articulation temporelle du biographique et du structurel. En: Revue Française de Sociologie. 35(2), pp. 283-291. Presses de Sciences Po (P.F.N.S.P.), París.

- Fernández Vavrik, G. (2016). "Un recién llegado y un pueblo kafkiano". En: Naishtat, F. (ed.) La crisis de sentido en debate. Historicidad, Subjetivación y Política. Biblos [en prensa], Buenos Aires.

- Freitas, A. A. \& Nunes, C. M. (noviembre, 2014). "Ser professor iniciante no contexto brasileiro: o que dizem as pesquisas" En: IV Jornadas Nacionales y II Latinoamericanas de Investigadores/as en Formación en Educación. 25, 26 y 27 de noviembre de 2014. Instituto de Investigaciones en Ciencias de la Educación. Facultad de Filosofía y Letras, Universidad de Buenos Aires. Buenos Aires. Recuperado http://iice.institutos.filo.uba.ar/webfm_send/7 [consultado 30/10/2018].

- Miyazaki (2015). "sado fue mejor? Un análisis de la formación y el desempeño de los ap opmeit odoTi (2013-nuevos docentes y sus representaciones en la prensa gráfica argentina (2000". En: Revista Mexicana de Investigación Educativa. 20(64), pp. 123-151. Recuperado http://www.comie.org.mx/v1/revista/portal.php?idm=es\&sec=SC03\&\&sub=SBB\&criterio=ART64006 [consultado 30/10/2018].

- Miyazaki (2017). "La formación docente puesta en cuestión. Relatos de los profesores principiantes de la escuela secundaria". En: Orce, V. (comp.) La educación como espacio de disputa. Miradas y experiencias de los/las investigadores/as en formación. Facultad de Filosofía y Letras. Universidad de Buenos Aires [en prensa], Buenos Aires.

- Miyazaki (2018). “UniCABA y Secundaria del Futuro: Dos caras de una misma moneda”. En: Observatorio Participativo de Políticas Públicas en Educación (OPPPEd), octubre 2018, 1-5. Facultad de Filosofía y Letras, Universidad de Buenos Aires. Recuperado http://iice.institutos.filo.uba.ar/webfm_send/108 
REVISTA DE LA ESCUELA de CIENCIAS DE LA EDUCACIÓN, AÑo 15, NRO. 14, VOL. 1, ENERO A JUNIO dE 2019. PÁGINAS 59-69. ISSN 1851-6297 (DESDE DICIEMBRE DE 2006 A DICIEMBRE DE 2017). ISSN 2362-3349 (EN LINEA). DE RELATOS Y EXPERIENCIAS. ELEGIR LA UNIVERSIDAD PARA FORMARSE COMO PROFESOR DE LA ESCUELA SECUNDARIA. ANDREA IGLESIAS.

[consultado 30/10/2018].

- Larrosa, J. (2003). "Algunas notas sobre la experiencia y sus lenguajes". Conferencia del Seminario Internacional "La Formación Docente entre el siglo XIX y el siglo XXI". Buenos Aires: Ministerio de Educación, Ciencia y tecnología/OEl. Recuperado de http://www.me.gov.ar/curriform/publica/oei_20031128/ponencia_larrosa.pdf [consultado 30/10/2018].

- Marcelo, C. (2012). "Empezar con buen pie: inserción a la enseñanza para profesores principiantes", Olhar de Professor. 15(2), pp. 209-221. Paraná, Brasil: Departamento de Métodos e Técnicas de Ensino. Recuperado de http://www.redalyc.org/pdf/684/68425573002.pdf [consultado 30/10/2018].

- Maxwell, J. A. (1996). Qualitative research design. An interactive approach. Sage Publications, Thousand Oaks.

- Mendizábal, N. (2006). "Los componentes del diseño flexible en la investigación cualitativa". En: Vasilachis de Gialdino, I. (coord.) Estrategias de investigación cualitativa (pp. 65-105). Gedisa, Barcelona.

- Menghini, R. A. y Negrin, M. (Comps.) (2015). Docentes principiantes. Aventuras y desventuras de los inicios en la enseñanza. Noveduc, Buenos Aires.

- Mereshián, N. R. y Calatayud, P. (2009). "Alternativas en la elección de la carrera docente". En: Educación, Lenguaje y Sociedad. 6(6), pp. 251-264. Recuperado de http://www. biblioteca.unlpam.edu.ar/pubpdf/ieles/n06a13mereshian.pdf [consultado 30/10/2018].

- Molinari, A. (2005). "La formación docente en la universidad". En: Cardinaux, N.; Clerico, L.; Molinari A. y Ruiz G. (Coords.) De cursos y de formaciones docentes. Historia de la carrera docente de la Facultad de Derecho de la UBA. Facultad de Derecho. Universidad de Buenos Aires, Buenos Aires.

- Muñiz Terra, L. (agosto, 2011). "Carreras y trayectorias laborales: aproximaciones teórico-metodológicas a su conceptualización, reconstrucción y análisis”. En: X Congreso Nacional de Especialistas en Estudios del Trabajo. ASET. 3 al 5 de agosto de 2011. Facultad de Ciencias Económicas (UBA). Buenos Aires. Recuperado de http://www.aset.org.ar/congresos/10/ponencias/p17_Muniz.pdf [consultado 30/10/2018].

- Muñoz, S. (2016). "Enseñar en escuelas secundarias. Recuperando perspectivas desde la formación docente de grado". En: Insaurralde, M. (Coord.) Enseñar en las Universidades y en los Institutos de Formación Docente. pp. 121-129. Noveduc, Buenos Aires.

- $\quad$ OEI (2010). Metas educativas 2021. La educación que queremos para la generación de los bicentenarios (Documento final). Organización de Estados Iberoamericanos para la Educación, la Ciencia y la Cultura (OEI), Madrid. Recuperado de http://www.oei.es/metas2021.pdf [consultado 1/10/2018]

- Périer, P. (2014). Professeurs débutants: les épreuves de l'enseignement. Presses Universitaires de France, Paris.

- Piovani, V. (2013). "Las políticas públicas de formación docente en la Argentina actual". En: Poggi, M. (coord.) Políticas docentes: formación, trabajo y desarrollo profesional (pp. 223-237). Instituto Internacional de Planeamiento de la Educación IIPE-Unesco. Buenos Aires. Recuperado http://www.buenosaires.iipe.unesco.org/sites/default/files/LibroPoliticas_docentes.pdf [consultado 30/10/2018].

- $\quad$ Rayou, P. \& Ria, L. (2009). "Former les nouveaux enseignants. Autour des statuts, de l'organisation et des saviors professionnels". En: Education et sociétés. 1(23), pp. 79-90. Recuperado de http://www.cairn.info/revue-education-et-societes-2009-1-page-79.htm [consultado 30/10/2018].

- $\quad$ Rinesi, E. (2015). Filosofía (y) política de la universidad. Ediciones UNGS, Buenos Aires.

- Soneira, A. J. (2006). "La teoría fundamentada en los datos (Grounded Theory) de Glaser y Strauss". En: Vasilachis de Gialdino, I. (coord.) Estrategias de investigación cualitativa (pp. 153-173). Gedisa, Buenos Aires.

- Southwell, M. y Vassiliades, A. (2014). "El concepto de posición docente: notas conceptuales y metodológicas". En: Educación, Lenguaje y Sociedad. 11(11), pp. 163-187. Instituto para el Estudio de la Educación, el Lenguaje y la Sociedad, Facultad de Ciencias Humanas, Universidad Nacional de La Pampa (FCH, UNLPam). Recuperado http://cerac.unlpam.edu.ar/index.php/els/article/view/1491 [consultado 30/10/2018].

- Tenti Fanfani, E. (2005). La condición docente. Análisis comparado de la Argentina, Brasil, Perú y Uruguay. IIPE/UNESCO/Fundación OSDE/Siglo XXI, Buenos Aires.

- Tenti Fanfani, E. (2010). Estudiantes y profesores de los IFD. Opiniones, valoraciones y expectativas. Documento preliminar. IIPE/UNESCO, Sede regional Buenos Aires. Buenos Aires/Ministerio de Educación de la Nación. Recuperado http://cedoc.infd.edu.ar/upload/documento_Tenti_Fanfani.pdf [consultado 30/10/2018]

- $\quad$ Tenti Fanfani, E. y Steinberg, C. (2011). Los docentes mexicanos. Datos e interpretaciones en perspectiva comparada. IIPE/UNESCO/Siglo XXI, Buenos Aires.

- Terigi, F. (coord.) (2011). Aportes pedagógicos a la reformulación de la formación inicial de los/as profesores/as de nivel secundario en Argentina. Informe final, noviembre 2011. Buenos Aires. Instituto Nacional de Formación Docente. Ministerio de Educación de la Nación. Presidencia de la Nación. Recuperado http://repositorio.educacion.gov.ar:8080/dspace/bitstream/handle/123456789/109613/06.\%20APORTES\% 20PEDAG\%C3\%93GICOS\%20A\%20LA\%20REFORMULACI\%C3\%93N\%20DE\%20LA\%20FORMACI\%C $3 \% 93 \mathrm{~N} \% 20 \mathrm{l}$.pdf?sequence=1 [consultado 30/10/2018].

- Vasilachis de Gialdino, I. (2006). "La investigación cualitativa". En: Estrategias de investigación cualitativa (23-64). Gedisa, Barcelona.

- Villanueva Gutiérrez, O. E. (2010). De estudiantes a profesores. Transiciones y dilemas en la incorporación profesional. Miguel Ángel Porrúa. México D.F. 